8 Corresponding Author:

$9 \quad$ Liam F. Fitzgerald

10 Department of Physical Therapy

111225 Center Drive, University of Florida

12 Gainesville, FL, USA, 32610

13 Email: 1.fitzgerald@phhp.ufl.edu

14

15 ORCID IDs:

16

17 Miles F. Bartlett: 0000-0001-7293-4214

18

19

20

21

22

23

24

Liam F. Fitzgerald: 0000-0002-8839-0001

Jules D. Miehm: 0000-0003-0651-3280

Jane A. Kent: 0000-0001-5487-2849

\title{
Effects of Old Age on Knee Extensor Fatigue and Recovery From High-Velocity Muscle Contractions
}

\author{
Liam F. Fitzgerald, Margaret M. Ryan, Miles F. Bartlett, Jules D. Miehm, Jane A. Kent
}

Muscle Physiology Lab, Department of Kinesiology, University of Massachusetts Amherst, USA

LFF and JAK conceived and designed research. LFF, MMR, MFB, and JDM collected the data.

22 LFF, MMR, and MFB analysed the data. LFF and JAK interpreted the data. The first draft of the manuscript was written by LFF and all authors commented on previous versions of the manuscript. All authors read and approved the final manuscript. 


\section{ABSTRACT}

Purpose: Although high-velocity contractions elicit greater muscle fatigue in older than young adults, the cause of this difference is unclear. We examined the potential roles of resting muscle architecture and baseline contractile properties, as well as changes in voluntary activation and low-frequency fatigue in response to high-velocity knee extensor work. Methods: Vastus lateralis muscle architecture was determined in quiescent muscle by ultrasonography in 8 young (23.4 \pm 1.8 yrs) and 8 older women (69.6 \pm 1.1$)$. Maximal voluntary dynamic (MVDC) and isometric (MVIC), and stimulated $(80 \mathrm{~Hz}$ and $10 \mathrm{~Hz}, 500 \mathrm{~ms})$ isometric contractions were performed before and immediately after $120 \mathrm{MVDCs}\left(240^{\circ} \cdot \mathrm{s}^{-1}\right.$, one every $\left.2 \mathrm{~s}\right)$. Results: Architecture variables did not differ between groups ( $\mathrm{p} \geq 0.209)$, but the half-time of torque relaxation $\left(\mathrm{T}_{1 / 2}\right)$ was longer in older than young women at baseline (151.9 $\pm 6.0 \mathrm{vs} .118 .8 \pm 4.4 \mathrm{~ms}$, respectively, $\mathrm{p}=0.001$ ). Older women fatigued more than young (to $33.6 \pm 4.7 \%$ vs. $55.2 \pm 4.2 \%$

37 initial torque, respectively; $p=0.004$ ), with no evidence of voluntary activation failure ( $\triangle$ MVIC:80Hz torque) in either group ( $\mathrm{p} \geq 0.317)$. Low-frequency fatigue $(\Delta 10: 80 \mathrm{~Hz}$ torque) occurred in both groups $(\mathrm{p}<0.001)$, as did slowing of $\mathrm{T}_{1 / 2}(\mathrm{p}=0.001)$, with no differences between groups. Baseline $T_{1 / 2}$ was inversely associated with fatigue in older $\left(r^{2}=0.584, p=0.045\right)$, but not

41 young women $\left(\mathrm{r}^{2}=0.147, \mathrm{p}=0.348\right)$. Conclusions: These results indicate that differences in

42 muscle architecture, voluntary activation, and low-frequency fatigue do not explain the greater

43 fatigue of older compared with young women during high-velocity contractions. The inverse

44 association between baseline $\mathrm{T}_{1 / 2}$ and fatigue in older women suggests that factors related to 45 slower muscle contractile properties may be protective against fatigue during fast, repetitive 46 contractions in aging. 
bioRxiv preprint doi: https://doi.org/10.1101/2020.05.22.110346; this version posted May 22, 2020. The copyright holder for this preprint (which

was not certified by peer review) is the author/funder, who has granted bioRxiv a license to display the preprint in perpetuity. It is made available under aCC-BY 4.0 International license.

47 Key Words: power, contractile properties, excitation-contraction coupling, muscle architecture,

48 low-frequency fatigue, neural activation 
Maximal torque and power production are lower in older compared with younger adults

52 [1-6], due to factors such as a loss of muscle mass [5,7-10], slower maximal contraction

53 velocities [1,2,11], and lower maximal motor unit discharge rates [12]. These changes have

54 significant consequences for older adults because deficits in muscular power are associated with an increased likelihood of falling, which may place older adults at risk of injury, poor mobility and subsequent loss of independence $[13,14]$. In particular, power production by the knee extensor muscles is important due to its association with physical function in aging [15], as well as the concept that rapid contraction of the knee extensors appears to be crucial for preventing

59 falls following a balance perturbation [16]. part, by changes in muscle architecture such as muscle thickness, pennation angle, and fascicle length [17]. Generally, muscles with a greater pennation angle have more sarcomeres in parallel, whereas muscles with longer fascicles contain more sarcomeres in series [18]. Conceptually, a greater number of sarcomeres in parallel allows for greater torque production, while more sarcomeres in series allows for greater velocity of shortening [19]. In aging, the loss of muscle mass results in architectural remodeling [20]; fascicles can

67 become shorter and less pennate [21]. Previous work has shown fascicle length and pennation angle of the vastus lateralis to be $\sim 12 \%$ shorter and $\sim 25 \%$ smaller, respectively, in older compared with young men [22]. Given the apparent lack of sex-related differences in the decline of quadriceps muscle volume with age [8], similar age-related changes in muscle architecture

71 may be expected in women. However, Kubo et al. observed an age-related decrease in fascicle 
72 length in the vastus lateralis in men but not women [23]. Changes in fascicle length may account

73 for $\sim 20 \%$ of the age-related difference in maximal shortening velocity of the medial

74 gastrocnemius in men [4]. Thus, age-related differences in fascicle length could place older

75 muscles at a disadvantage for generating power during high-velocity contractions [5,11,24-26].

76 To date, the extent to which architectural remodeling affects age-related differences in muscle

77 fatigue is not known.

Muscle fatigue is defined as the reduced capacity to produce torque or power in response

to a period of contractile activity [27]. Mechanisms of fatigue can include transient impairments at any site along the pathway of force or power production, from the brain to the myofilaments

[27]. The literature generally supports the observation of less muscle fatigue in older ( $\geq 65$ years) compared with young ( $\leq 40$ years) adults in response to isometric contractions [28]. Conversely, during dynamic contractions at high velocities, or when fatigue is expressed as a decline in power, older adults fatigue to a greater extent than young adults [5,11,24-26,29]. Despite consistent observations of these age-related differences in muscle fatigue, the mechanisms for greater fatigue in older compared with young adults in response to high-velocity contractions remain poorly understood.

Use of percutaneous stimulation offers a non-invasive approach for identifying whether the cause of fatigue is proximal or distal to the stimulating electrodes (i.e., central or peripheral) [30]. Small reductions $(\sim 5 \%)$ in voluntary activation, defined as the neural drive to the contracting muscle, have been observed in both young and older men following contractions of the knee extensors at slow- and moderate-velocities [24]. More recently, Sundberg et al. observed failure of voluntary activation in older, but not young, women following 80 maximalvelocity contractions at a load of $20 \%$ maximal isometric torque [29]. However, the magnitude 
of the change in voluntary activation in the older women was small $(<2 \%)$, and no association was observed between fatigue ( $\%$ initial power) and changes in voluntary activation. Thus, agerelated differences in voluntary activation failure do not appear to contribute to age-related differences in fatigue in response to high-velocity contractions.

The presence of low-frequency fatigue (LFF) can also be evaluated in vivo using myoelectric stimulation by comparing fatigue-induced changes in torque in response to low- vs. high-frequency stimuli [31-34]. With LFF, the decline and slowed recovery of torque produced by low-frequency stimuli are more pronounced than those elicited at higher frequencies [31]. Because LFF may stem, at least in part, from a failure in excitation contraction coupling (ECC) the low:high frequency torque ratio has been used as an indirect measure of ECC failure in vivo. In single muscle fibers, a greater loss of force in response to low- vs. high-frequency stimuli is associated with impaired $\mathrm{Ca}^{2+}$ release $[35,36]$. Thus, age-related impairments in $\mathrm{Ca}^{2+}$ handling could contribute to the development of LFF [37]. In response to fatiguing isometric contractions in humans, there appear to be no age-related differences in LFF [32,33]. However, the potential role of LFF in the greater fatigue and delayed recovery of power in older compared with younger adults in response to high-velocity contractions is not known.

In unfatigued muscle, the half-time of torque relaxation $\left(T_{1 / 2}\right)$ is generally longer in older than young adults $[5,24,25,29,38]$. This difference could result from greater type 1 fiber composition [39] or differences in cross-bridge kinetics [40] in older muscle; the source of this slowing has not been definitively identified. In fatigue, $\mathrm{T}_{1 / 2}$ lengthens $[38,41]$, presumably due to a slowing of $\mathrm{Ca}^{2+}$ release from troponin or its re-uptake by the sarcoplasmic reticulum [42] in response to the accumulation of $\mathrm{H}^{+}$[43]. During fatiguing isometric contractions, some authors report a similar slowing of $\mathrm{T}_{1 / 2}$ in young and older muscle $[25,38,41]$. In contrast, during 
dynamic contractions of the knee extensor muscles, others reported a greater slowing of $\mathrm{T}_{1 / 2}$ in older compared with young men following contractions at moderate and fast, but not slow, velocities [24]. More recently, intracellular $\mathrm{pH}$ was shown to be lower in older than young muscle in response to dynamic contractions of the knee extensors to fatigue [44], which could contribute to a greater slowing of $\mathrm{T}_{1 / 2}$ in older than young muscle.

Given the existing questions about the causes of muscle fatigue in aging, the purpose of this study was to compare in young and older women several potential mechanisms that may contribute to age-related differences in fatigue following high-velocity concentric contractions of the knee extensor muscles. Baseline muscle architecture, as well as changes in voluntary activation and contractile properties, were evaluated during fatigue and for 30 minutes of recovery. We hypothesized that fatigue (fall of peak power relative to baseline) would be greater, and recovery of power slower, in older compared with young muscle. We further hypothesized that vastus lateralis fascicle length and pennation angle would be shorter and smaller, respectively, in older compared with young women, and that fascicle length would be positively associated with peak power production at baseline, given the importance of the number of sarcomeres in series for power generation [4]. Based on the current literature, we did not expect to observe any age-related differences in voluntary activation at the end of the fatigue protocol $[24,29]$. However, we did anticipate greater low-frequency fatigue and slowing of $\mathrm{T}_{1 / 2}$ in older compared with young women.

\section{METHODS}

Participants. Sample size estimates were calculated a priori to detect significant differences in fatigue ( $\%$ initial torque) with $80 \%$ power, an alpha level of 0.05 , and an equal 
number of participants in young and older groups (GPower v3.1; [45]). For this calculation, values from a study that used a similar fatigue protocol were used to determine the effect size [5]. These calculations revealed 8 participants were required per group to detect significant agerelated differences in muscle fatigue. Thus, 8 young (21-36 years) and 8 older (66-76 years) women were studied. Prior to enrollment, each participant gave their written, informed consent, as approved by the University of Massachusetts Amherst, and in accordance with the Declaration of Helsinki. Physician's approval to participate was obtained for all of the older volunteers between Visits 1 and 2. To avoid any potential age-by-sex interactions, we restricted this study to women. Participants reported being relatively sedentary in that they completed less than two 30-min sessions of structured exercise per week. They also reported being healthy, as evaluated by a health-history questionnaire, and were not taking any medications known to affect physical function or muscle fatigue (e.g., beta-blockers, calcium channel blockers, etc.). All participants answered 'no' to all questions on the Physical Activity Readiness Questionnaire [46]. To minimize the effect of fluctuating hormone levels on muscle function over the course of the menstrual cycle [47], all young women were studied during days 1-5 of the menses. No participants had used any hormone replacement therapies in the 6 months prior to enrolling in the study.

Procedures. Participants reported to the Muscle Physiology Laboratory for 2 Visits. At Visit 1, mobility function was measured and familiarization with the fatigue protocol was provided. During Visit 2, muscle architecture of the vastus lateralis was measured, followed by the knee extensor fatigue protocol. Visits were separated by at least 2 days.

Mobility function and activity. Measures of mobility and physical activity were obtained in order to characterize the study groups. Mobility was measured using the timed up-and-go 
164 (TUG; [48]) and advanced short physical performance battery (SPPB-A; [49]). The fastest time

165 for the TUG was used for analysis. At the end of Visit 1, participants were asked to wear a

166 uniaxial accelerometer (GT3X, Actigraph, Pensacola, FL) at the hip for 7 days in order to

167 characterize habitual physical activity levels. Participants wore the accelerometer for a

168 minimum of 10 hours per day, and at least 4 days ( 3 weekdays and 1 weekend day). These

169 criteria have been shown to provide good reliability $(>80 \%)$ for quantifying overall and

170 moderate-to-vigorous physical activity [50,51]. Average daily activity counts and minutes spent

171 in moderate-to-vigorous physical activity were calculated using ActiLife v6.13 software

172 (ActiGraph, Pensacola, FL) with established cutpoint thresholds [52].

Muscle architecture. Thigh, shank, and total leg length were measured using a tape

174 measure. The midpoint between the greater trochanter and lateral femoral condyle of the femur

175 was marked with indelible ink and thigh circumference was measured at this position.

176 Subsequently, the participant was seated on a Biodex System 3 dynamometer (Biodex Medical

177 Systems, Shirley, NY) with the same hip and knee angles used at Visit 1. After 10 min of seated

178 rest to account for fluid shifts in the thigh [53], ultrasound images of the participant's vastus

179 lateralis were collected via a linear array probe (Philips model L12-5, 12-5MHz), using a Philips

180 HD11XE system (Philips Healthcare, Bothell, WA). The probe was placed over the mark on the

181 participant's thigh, at approximately $50 \%$ of the length of the femur. Once the probe placement

182 was optimized, determined as the position that yielded the best visualization of the deep and

183 superficial aponeuroses and muscle fascicles, 5 images were collected. All images were

184 obtained at a hip and knee angle of $90^{\circ}$ and $100^{\circ}$, respectively. To check whether peripheral

185 edema occurred during the imaging session, the widest circumference of the calf was measured

186 before and after images were obtained. 
measured as the distance between the internal borders of the superficial and deep aponeuroses.

192 follows:

$$
\text { Estimated fascicle length }=\frac{\text { Muscle thickness }}{\operatorname{Sin}(\theta)}
$$

where $\Theta$ represents the measured pennation angle. This approach, illustrated in Fig 1, is

Fig 1. Ultrasound image from a representative young woman showing estimated fascicle length (eFL), muscle thickness (MT), and pennation angle $(\Theta)$.

Muscle torque. Prior to the measurement of isometric and isokinetic torque, participants completed 5 min of unloaded cycling at $\sim 60$-rpm to warm-up. The dynamometer was used to test knee extensor torque during maximal voluntary isometric (MVIC) and dynamic (MVDC) contractions of the dominant leg (preferred kicking leg), respectively. Participants were seated 
upright with their hips at $90^{\circ}$ and a knee angle of $100^{\circ}$ extension $\left(180^{\circ}=\right.$ full extension $)$. The dynamometer was adjusted to ensure that the axis of rotation of the dynamometer's lever arm was aligned with the axis of rotation of the participant's knee joint. The length of the lever arm was adjusted and secured with inelastic straps proximal to the malleoli. Additional straps were fastened across the participant's torso and hips to prevent unwanted movement. Participants were instructed not to hold onto any part of the dynamometer during the testing to ensure that torque and velocity measurements were generated by the knee extensor muscles.

Peak knee extensor torque was measured with the dynamometer in isometric and isokinetic modes to determine MVIC and MVDC at $240^{\circ} \cdot \mathrm{s}^{-1}$, respectively. To determine the target torque for electrically-stimulated contractions (see Myoelectric stimulation below), participants completed 2 brief MVICs at a knee angle of $100^{\circ}$ with 1 min rest between contractions. If those 2 MVICs differed by more than $10 \%$, additional MVICs were completed until the 2 strongest contractions were within $10 \%$ of each other. To ensure participants were still familiar with the dynamic contractions performed at visit 1, participants completed two sets of 2 MVDCs at a concentric velocity of $240^{\circ} \cdot \mathrm{s}^{-1}$, with 1 min rest between sets to prevent muscle fatigue. Participant's limbs were allowed to return to the start position at a velocity of $500^{\circ} \cdot \mathrm{s}^{-1}$ (i.e., against no load), and all MVDCs were completed over a $70^{\circ}$ range of motion. For all contractions, the participant sat with her arms folded across her chest and were instructed to "kick your leg out as hard and fast as possible." Visual torque-feedback, scaled to each participant's maximum torque, was provided during each contraction.

Analog signals corresponding to torque, velocity, and position were acquired from the dynamometer at a sampling rate of 2,500 Hz. Data were analyzed as reported previously $[1,5,41,55]$. Briefly, peak torque was taken during the target isovelocity period. 

muscle contractile properties during isometric contractions at baseline, immediately after the fatigue protocol, and at 5,10, 20, and $30 \mathrm{~min}$ of recovery. Two $7.6 \times 12.7 \mathrm{~cm}$ self-adhesive

235 stimulating electrodes were placed transversely across the thigh. One electrode was positioned

$236 \quad 3-5 \mathrm{~cm}$ distal to the inguinal crease and the other was placed 2-3 $\mathrm{cm}$ proximal to the superior

237 border of the patella. High- $(80-\mathrm{Hz})$ and low-frequency $(10-\mathrm{Hz})$ stimulations were delivered

238 (200- $\mu$ s pulse duration, $500 \mathrm{~ms}$ ) with a constant-current stimulator (DS7AH; Digitimer,

239 Hertfordshire, UK). The current that elicited an $80-\mathrm{Hz}$ contraction resulting in $50 \%$ of the

240 participant's MVIC was used for all subsequent stimulations, as described previously [5,55].

241 Immediately prior to the fatigue protocol, participants completed a series of voluntary and

242 electrically-stimulated contractions consisting of $2 \mathrm{MVDCs}\left(240^{\circ} \cdot \mathrm{s}^{-1}\right), 1 \mathrm{MVIC}$, one $80-\mathrm{Hz}$ and

243 one $10-\mathrm{Hz}$ contraction. A representative torque trace from the baseline series of contractions for

2441 young woman is shown in Fig 2. This series of contractions was repeated at the end of the

245 fatigue protocol and throughout recovery.

247 Fig 2. Representative baseline series of contractions. Data are from one young woman

Muscle contractile properties were quantified as peak torque during the 10 and $80-\mathrm{Hz}$

252 account for the effects of peak torque achieved on this measure. The $\mathrm{T}_{1 / 2}$ was measured as the

253 time for torque to decline to $50 \%$ of that achieved at the time of the last stimulus during the 80-

$254 \mathrm{~Hz}$ contraction. Changes in the ratio of peak torques elicited during the $10-\mathrm{Hz}$ and $80-\mathrm{Hz}$ 
stimulations was used to determine the presence of LFF [31]. Additionally, the ratio of MVIC:80-Hz torque was calculated at each time point. A decrease in this ratio reflects a greater fall in voluntary than stimulated torque production, suggesting the presence of central fatigue $[56]$.

Fatigue protocol. Following the baseline test series of contractions, the participant completed a unilateral knee extension fatigue protocol consisting of $1 \mathrm{MVDC}$ at $240^{\circ} \cdot \mathrm{s}^{-1}$ every 2 $\mathrm{s}$ for $4 \mathrm{~min}$. Each contraction was cued by an auditory signal and strong verbal encouragement was provided by the investigators throughout the protocol. Visual torque feedback, scaled to each participant's MVIC, was provided throughout the fatigue protocol and recovery. Fatigue was calculated as [5]:

$$
\frac{\text { (average peak torque of the final } 5 \text { contractions) }}{\text { IVerage of peak torque at baseline and during the fatigue trial) }} \times 100
$$

To evaluate potential sites of fatigue and the recovery of MVDC torque following fatigue, the test series was repeated immediately after the final contraction of the fatigue trial, and at 5, 10, 20 , and $30 \mathrm{~min}$ of recovery.

Statistical analyses. All data were checked for normality and homogeneity of variance prior to any statistical comparisons, using the Kolmogorov-Smirnov test and Levene's statistic, respectively. If the assumptions of normality or homogeneity of variance were violated, nonparametric statistical comparisons were performed. Differences in group descriptive characteristics, muscle architecture, and fatigue were analyzed using independent t-tests or Mann-Whitney U tests. Changes in peak MVDC torque, MVIC:80-Hz torque, 10:80-Hz torque, and contractile properties (RTD and $\mathrm{T}_{1 / 2}$ ) between baseline and fatigue, and throughout recovery, were analyzed using separate 2-way repeated measures ANOVAs (group $\times$ time). Linear regression analyses were performed to evaluate the relationships between muscle architecture 
277 and baseline MVIC and MVDC torque, as well as fatigue and measures of physical activity and

278 function, muscle architecture, and contractile properties. For regression analyses in which

279 estimated fascicle length was used, only the non-normalized values were included. All statistical

280 analyses were performed using the SPSS statistical software package (v25.0, IBM, Chicago, IL)

281 with an alpha level of 0.05 . Data are reported as mean \pm SE, with exact $p$-values, $95 \%$ confidence

282 intervals for differences between group means, and effect sizes also provided. Effect sizes were

283 calculated as Cohen's d [57].

\section{RESULTS}

Baseline characteristics. Group characteristics are reported in Table 1. The young and

287 older groups had similar height, body mass, and BMI. The young group had greater habitual 288 physical activity (daily counts and MVPA) compared with the older group. The measures of 289 mobility function indicated a difference only for the SPPB-A, which was lower in the older 290 group.

Table 1. Group characteristics

\begin{tabular}{lccccc}
\hline & Young $(\mathrm{n}=8)$ & Older $(\mathrm{n}=8)$ & $95 \%$ CI & $P$ & Effect size \\
\hline Age $(\mathrm{yr})$ & $23.4 \pm 1.8$ & $69.6 \pm 1.1$ & $-50.8,-41.6$ & & \\
Height $(\mathrm{cm})$ & $164.5 \pm 1.9$ & $164.3 \pm 2.6$ & $-6.6,7.1$ & 0.942 & 0.03 \\
Body mass $(\mathrm{kg})$ & $65.3 \pm 5.9$ & $66.4 \pm 3.8$ & $-16.2,14.0$ & 0.877 & 0.08 \\
BMI $\left(\mathrm{kg} \cdot \mathrm{m}^{-2}\right)$ & $24.1 \pm 2.2$ & $24.6 \pm 1.3$ & $-6.0,5.0$ & 0.851 & 0.10 \\
PA $\left(\mathrm{counts} \cdot \mathrm{d}^{-1000}\right)$ & $286.0 \pm 29.2$ & $162.6 \pm 10.4$ & $52.8,193.8$ & 0.005 & 1.99 \\
MVPA $\left(\mathrm{min} \cdot \mathrm{d}^{-1}\right)$ & $47.3 \pm 6.5$ & $11.2 \pm 2.3$ & $20.5,51.8$ & 0.001 & 2.62 \\
Gait speed $\left(\mathrm{m}^{-2} \mathrm{~s}^{-2}\right)$ & $1.20 \pm 0.04$ & $1.17 \pm 0.07$ & $-0.1,0.2$ & 0.740 & 0.18
\end{tabular}




$\begin{array}{lccccr}\text { Chair rise time (s) } & 14.1 \pm 1.1 & 19.5 \pm 1.6 & -9.5,-1.3 & 0.140 & 1.41 \\ \text { TUG (s) } & 8.3 \pm 0.2 & 9.0 \pm 0.6 & -2.1,0.7 & 0.382 & 0.59 \\ \text { SPPB-A } & 2.9 \pm 0.1 & 2.5 \pm 0.1 & 0.02,0.6 & 0.037 & 1.57\end{array}$

Values are mean \pm SE. $95 \%$ CI, $95 \%$ confidence intervals for the difference between group means;

BMI, body mass index; PA, physical activity; MVPA, moderate- to vigorous-intensity physical activity; TUG, timed up-and-go; SPPB-A, advanced short physical performance battery.

Table 2. Effects of aging on properties of the vastus lateralis

\begin{tabular}{|c|c|c|c|c|c|}
\hline & Young $(n=8)$ & Older $(n=8)$ & $95 \% \mathrm{CI}$ & $P$ & Effect size \\
\hline \multicolumn{6}{|l|}{ Muscle architecture } \\
\hline Pennation angle $\left(^{\circ}\right)$ & $13.5 \pm 0.4$ & $13.0 \pm 0.6$ & $-1.1,2.1$ & 0.524 & 0.33 \\
\hline MT (mm) & $26.1 \pm 1.5$ & $23.0 \pm 1.8$ & $-2.0,8.1$ & 0.209 & 0.66 \\
\hline eFL (mm) & $113.7 \pm 8.8$ & $102.7 \pm 8.0$ & $-14.5,36.5$ & 0.371 & 0.46 \\
\hline eFL/thigh length & $3.1 \pm 0.3$ & $2.7 \pm 0.2$ & $-0.4,1.1$ & 0.330 & 0.50 \\
\hline \multicolumn{6}{|l|}{ Contractile properties } \\
\hline $\operatorname{MVDC}(\mathrm{Nm})$ & $84.0 \pm 7.1$ & $49.2 \pm 6.0$ & $14.9,54.7$ & 0.002 & 1.87 \\
\hline MVIC (Nm) & $134.7 \pm 12.0$ & $93.8 \pm 6.5$ & $11.7,70.3$ & 0.01 & 1.50 \\
\hline RTD $\left(\% p k \cdot m s^{-1}\right)$ & $1.33 \pm 0.12$ & $1.08 \pm 0.10$ & $-0.1,0.6$ & 0.119 & 0.83 \\
\hline $\mathrm{T}_{1 / 2}(\mathrm{~ms})$ & $118.8 \pm 4.4$ & $151.9 \pm 6.0$ & $-49.0,-17.2$ & 0.001 & 2.20 \\
\hline
\end{tabular}


Values are mean \pm SE. 95\% CI, 95\% confidence intervals for differences in group means; MT, muscle thickness; eFL, estimated fascicle length; MVDC, maximal voluntary dynamic contraction; MVIC, maximal voluntary isometric contraction; $\mathrm{RTD}$, maximum rate of torque development; $\mathrm{T}_{1 / 2}$, torque half-relaxation time.

The within-subject coefficient of variation for the 3 images analyzed for pennation angle,

297

298

299

300

301

302

muscle thickness, and estimated fascicle length were $5.7 \pm 1.2$ and $5.0 \pm 1.1,3.9 \pm 1.3$ and $3.8 \pm 0.9$, and $5.7 \pm 1.5$ and $5.2 \pm 1.3 \%$ for young and older women, respectively, indicating that these were robust measurements in both groups. Calf circumference did not change from pre- to postimaging in young $(38.8 \pm 1.6$ vs. $38.8 \pm 1.5, \mathrm{p}=0.991)$ or older $(36.4 \pm 1.4$ vs. $36.5 \pm 1.4, \mathrm{p}=0.950)$ women, suggesting that fluid shifts did not impact the architecture measures. There were no differences between groups in pennation angle, muscle thickness, or fascicle length (Table 2), nor was peak MVDC torque at baseline associated with fascicle length in young $\left(\mathrm{r}^{2}=0.098\right.$, $\mathrm{p}=0.449)$ or older women $\left(\mathrm{r}^{2}=0.148, \mathrm{p}=0.393\right)$. Neither baseline peak MVDC torque nor maximal isometric torque were associated with pennation angle or muscle thickness in young women $\left(\mathrm{r}^{2} \leq 0.083, \mathrm{p} \geq 0.49\right)$. Likewise, neither peak MVDC torque nor maximal isometric torque were associated with pennation angle or muscle thickness in older women $\left(r^{2} \leq 0.143, p \geq 0.403\right)$. Lastly, muscle thickness was positively associated with maximal isometric torque in older women $\left(\mathrm{r}^{2}=0.613, \mathrm{p}=0.022\right)$.

Muscle fatigue. Changes in peak torque during the 4-min fatigue protocol are shown in Fig 3. With the exception of 1 older woman, who could not attain target velocity after the first 25 contractions, all participants were able to complete the 4-min fatigue protocol. Therefore, the fatigue data reported here represent the average for 7 older women. Fatigue was greater in older compared with young women $(33.6 \pm 4.7$ vs. $55.2 \pm 4.2 \%$ initial torque, respectively; $p=0.004,95 \%$ 
CI: 8.0-35.1, $\mathrm{d}=1.85$ ). Analysis of changes in the MVIC:80-Hz torque ratio (from $2.02 \pm 0.06$ and $1.87 \pm 0.11$ at baseline in young and older groups, respectively, to $1.94 \pm 0.10$ and $1.77 \pm 0.09$ in young and older at fatigue) revealed no age $\times$ time interaction $(\mathrm{p}=0.918)$ or main effects of group $(\mathrm{p}=0.097)$ or time $(\mathrm{p}=0.317)$, indicating no failure of voluntary activation in either group in response to fatiguing contractions.

Fig 3. Age-related differences in muscle fatigue and recovery. Older women experienced greater fatigue $(* p=0.005)$. There was a group $\times$ time interaction $(\dagger p=0.03)$ for recovery of peak torque over the first $5 \mathrm{~min}$, but both groups fully recovered within $5 \mathrm{~min}$ following contractions. Data are mean \pm SE. The dark and light grey shaded boxes represent the contraction protocol and

325 recovery period, respectively.

$332(\mathrm{p}=0.001$, Fig $4 \mathrm{~B})$. The RTD did not change with fatigue in young $(\mathrm{p}=0.783)$ or older groups $(p=0.99$, data not shown).

Fig 4. Fatigue and recovery of contractile properties in young and older women. A) 10:80-Hz torque at baseline, fatigue, and throughout recovery. The 10:80-Hz torque ratio decreased with

337 fatigue (main effect of time: $\# p=0.001$ ) and increased over the first 5 minutes of recovery in both 
groups (main effect of time: $\# \mathrm{p} \leq 0.01$ ). B) $\mathrm{T}_{1 / 2}$ at baseline, fatigue, and throughout recovery.

Relaxation was slower in older compared with young women at baseline, fatigue, and following 5 minutes of recovery (main effect of group: ${ }^{*} \mathrm{p} \leq 0.04$ ). In both groups, $\mathrm{T}_{1 / 2}$ slowed with fatigue and recovered within 5 minutes of recovery in both groups (main effect of time: $\# p=0.001$ ), with no groupxtime interactions. Data are mean \pm SE. The dark and light grey shaded boxes represent the contraction protocol and recovery period, respectively.

interaction $(\mathrm{p}=0.03)$ such that older women recovered more in the first 5 min following the protocol than young women $(\mathrm{p}=0.001$; Fig 3$)$. Peak MVDC torque did not differ from baseline by $5 \mathrm{~min}$ of recovery in either group. Maximal isometric torque recovered following the fatigue protocol (main effect of time, $\mathrm{p}=0.001$ ), with no effect of group $(\mathrm{p}=0.95)$ and no group $\times$ time interaction $(\mathrm{p}=0.103$, Table 3$)$. Full recovery of MVIC was observed 5 min post-fatigue in young and older groups $(\mathrm{p} \leq 0.001)$.

Table 3. Recovery of voluntary and stimulated isometric torque production

$\begin{array}{lllll}\text { Young }(\mathrm{n}=8) & \text { Older }(\mathrm{n}=8) & 95 \% \mathrm{CI} & P & \text { Effect size }\end{array}$

\begin{tabular}{llllll}
\hline \multicolumn{2}{l}{ MVIC (\% baseline) } & & & \\
0R & $76.3 \pm 4.0$ & $68.5 \pm 1.7$ & $1.3,14.3$ & 0.019 & 0.89 \\
$5 \mathrm{R}$ & $95.6 \pm 2.0$ & $96.0 \pm 1.7$ & $-6.8,6.2$ & 0.921 & 0.08 \\
$10 \mathrm{R}$ & $94.6 \pm 2.3$ & $97.4 \pm 1.2$ & $-9.3,3.8$ & 0.403 & 0.53 \\
20R & $91.7 \pm 3.5$ & $95.8 \pm 1.9$ & $-10.7,2.4$ & 0.210 & 0.51 \\
$30 \mathrm{R}$ & $94.7 \pm 1.8$ & $94.9 \pm 1.0$ & $-6.6,6.4$ & 0.976 & 0.05 \\
$80 \mathrm{~Hz}(\%$ baseline) & & & & &
\end{tabular}




$\begin{array}{cccccc}\text { 0R } & 79.6 \pm 2.8 & 72.4 \pm 3.1 & -3.6,17.9 & 0.191 & 0.86 \\ \text { 5R } & 83.4 \pm 3.7 & 78.6 \pm 4.0 & -6.0,15.5 & 0.381 & 0.44 \\ \text { 10R } & 77.6 \pm 4.3 & 72.0 \pm 3.6 & -5.1,16.4 & 0.301 & 0.50 \\ \text { 20R } & 77.7 \pm 4.3 & 70.6 \pm 3.5 & -3.7,17.8 & 0.196 & 0.64 \\ \text { 30R } & 80.3 \pm 4.1 & 68.2 \pm 4.4 & 1.3,22.8 & 0.029 & 1.01\end{array}$

\section{$10 \mathrm{~Hz}$ (\% baseline)}

$\begin{array}{llllll}\text { 0R } & 58.9 \pm 6.4 & 51.6 \pm 3.1 & -4.1,18.5 & 0.208 & 0.51 \\ 5 \mathrm{R} & 77.5 \pm 3.0 & 81.7 \pm 3.7 & -15.6,7.1 & 0.458 & 0.44 \\ \text { 10R } & 63.5 \pm 4.3 & 65.3 \pm 2.5 & -13.1,9.5 & 0.750 & 0.18 \\ \text { 20R } & 56.1 \pm 4.9 & 62.8 \pm 3.1 & -18.0,4.6 & 0.240 & 0.57 \\ \text { 30R } & 60.5 \pm 4.6 & 61.3 \pm 2.7 & -12.1,10.6 & 0.895 & 0.07\end{array}$

Values are mean \pm SE. 95\% CI, 95\% confidence intervals for differences in group means; MVIC, maximal voluntary isometric contraction; $80 \mathrm{~Hz}$, high-frequency stimulation; $10 \mathrm{~Hz}$, low-frequency stimulation.

354 observe main effects of time $(\mathrm{p}<0.001)$ and group $(\mathrm{p}=0.037$, Fig $4 \mathrm{~A})$ during recovery. The $35510: 80-\mathrm{Hz}$ torque ratio increased to pre-fatigue levels within $5 \mathrm{~min}$ of recovery in both groups $356(p \leq 0.01)$. There was no group $\times$ time interaction $(p=0.359)$, but we did observe main effects of 357 time $(\mathrm{p}<0.001)$ and group $(\mathrm{p}<0.001)$ for changes in $\mathrm{T}_{1 / 2}$ during recovery such that torque 358 relaxation recovered similarly in young and older women (Fig 4B). There was no group $\times$ time 359 interaction $(p=0.973)$ and no main effects of group $(p=0.085)$ or time $(p=0.867)$ for the RTD 360 during recovery (data not shown). 
Factors associated with fatigue. Regression analyses revealed that fatigue was not

362

363

364

365

366

367

368

369

370

371

372

373

374

375

376

377

378

379

associated with any parameter of muscle architecture in the young or older women $\left(\mathrm{r}^{2} \leq 0.14\right.$, $\mathrm{p} \geq 0.462$ ). The $\triangle \mathrm{MVIC}: 80-\mathrm{Hz}$ torque from baseline to post-fatigue was not associated with fatigue in young $\left(\mathrm{r}^{2}=0.17, \mathrm{p}=0.305\right)$ or older women $\left(\mathrm{r}^{2}=0.001, \mathrm{p}=0.995\right)$, confirming that voluntary activation failure did not contribute to fatigue in this study. The $\Delta 10: 80-\mathrm{Hz}$ torque from baseline to post-fatigue was directly associated with fatigue in young $\left(r^{2}=0.713, p=0.008\right)$ but not older women $\left(\mathrm{r}^{2}=0.012, \mathrm{p}=0.815\right)$. In contrast, baseline $\mathrm{T}_{1 / 2}$ was inversely associated with fatigue in older $\left(\mathrm{r}^{2}=0.584, \mathrm{p}=0.045\right)$ but not young women $\left(\mathrm{r}^{2}=0.147, \mathrm{p}=0.348\right)$, as illustrated in Fig 5. Neither physical activity counts nor MVPA were associated with fatigue in young $\left(\mathrm{r}^{2} \leq 0.034, \mathrm{p} \geq 0.661\right)$ or older $\left(\mathrm{r}^{2} \leq 0.053, \mathrm{p} \geq 0.618\right)$ women. Fatigue was associated with SPPB-A score in older $\left(\mathrm{r}^{2}=0.565, \mathrm{p}=0.051\right)$, but not younger $\left(\mathrm{r}^{2}=0.032, \mathrm{p}=0.673\right)$ women.

Fig 5. Associations between half relaxation time and fatigue. Fatigue (peak torque, $\%$ initial) was plotted against half relaxation time $\left(\mathrm{T}_{1 / 2}\right)$ at baseline. Linear regression analysis revealed an association between these variables for older $\left(r^{2}=0.584, p=0.045\right)$, but not young women $\left(\mathrm{r}^{2}=0.147, \mathrm{p}=0.348\right)$, suggesting the age-related slowing of $\mathrm{T}_{1 / 2}$ may prevent excessive muscle fatigue in older women.

\section{DISCUSSION}

This study was designed to evaluate whether age-related differences in muscle architecture, voluntary activation, or contractile properties might be potential mechanisms for the greater muscle fatigue observed in older adults in response to high-velocity muscle contractions.

Peak MVDC torque fell significantly more in healthy older women than in healthy young 
women, demonstrating the expected age-related difference in knee extensor muscle fatigue at high contraction velocities. The results indicate that baseline differences in contractile properties, and not acute changes in these properties in response to fatigue, may contribute to high-velocity fatigue in aging: baseline $\mathrm{T}_{1 / 2}$ was slower in older than young, and predictive of fatigue only in the older group. There were no age-related differences in muscle architecture across our study groups, and we provide new information that architecture did not contribute to the greater fatigue observed in the older group. Comparisons of the fall in voluntary vs. stimulated tetanic contractions during fatigue (i.e., MVIC: $80 \mathrm{~Hz}$ torque ratio) indicated no decrement in voluntary activation in either group in response to this high-velocity contraction protocol, ruling out activation failure as a source of the difference in muscle fatigue between age 394 groups. Comparable LFF was observed in both groups, indicating significant peripheral fatigue in response to this protocol that was independent of age. Overall, these results support previous reports of greater fatigue in older muscle during high-velocity work [5,24,29], and suggest that this difference may be due in part to chronic changes in muscle properties, but not architecture, in the aged.

Baseline. Notably, there were no age-related differences in baseline muscle architecture of the vastus lateralis in these groups of healthy women (Table 2). While some studies have 401 reported remodelling of muscle architecture in old age [4,20-22] such that muscle thickness, 402 pennation angle, and fascicle length are reduced with age, most of those studies were conducted 403 in men. In contrast to those results, our data are in agreement with those of Kubo et al. who 404 likewise observed no age-related differences in vastus lateralis fascicle length, normalized to 405 thigh length, in women [58]. However, our results differ from that study insofar as we observed 406 no age-related differences in pennation angle and muscle thickness. The cause of this 
407

408

409

410

411

412

413

414

415

416

417

418

419

420

421

422

423

424

425

426

427

428

429

discrepancy is not clear at present, but does not appear to be due to differences in the age of the participants, nor the repeatability of the measurement given the low coefficient of variation found in our study. Questions as to how factors such as habitual physical activity and sex may influence architectural changes in old age, as well as the potential functional impacts of these changes, remain to be answered.

At baseline, peak MVDC and MVIC torque were $\sim 40$ and $\sim 30 \%$ lower, respectively, in older compared with young women (Table 2), which is consistent with previous reports $[5,55,59]$. The maximal RTD during electrically-evoked contractions was not different between young and older groups, similar to some $[5,55]$ but not all reports in the literature [29]. The $\mathrm{T}_{1 / 2}$ was slower at baseline in older compared with young muscle, a result that is in agreement with most studies $[5,24,25,55]$, and potentially due to slowed $\mathrm{Ca}^{2+}$ dissociation from troponin, slower crossbridge kinetics [40], a greater relative proportion of type I fibers [9], or a decrease in muscle-tendon unit stiffness with age [60]. The slower relaxation in older muscle does not appear to be caused by slowed $\mathrm{Ca}^{2+}$ re-uptake by the sarcoplasmic reticulum with age [61] Fatigue. Consistent with previous reports [5,24,25,29], we observed greater knee extensor muscle fatigue in older compared with young women during contractions at $240^{\circ} \cdot \mathrm{s}^{-1}$. The question of whether older muscle fatigues more than young depends upon the contraction velocity used to fatigue the muscle, with greater fatigue reported at higher speeds, i.e., those to the right on the torque-velocity curve [5]. The fatigue resistance of older muscle under isometric conditions is well reported [28], and the cause of this difference appears to be due to a lower reliance on glycolytic ATP production in older compared with young muscle [62,63]. Fatigue induced by slow- and moderate-velocity dynamic contractions is similar between young and older adults $[5,24,55]$, while fatigue is generally greater in older than young muscle in response 
to high-velocity contractions $[5,24-26,29,44]$, as found here. The goal of this study was to

431

432

433

434

435

436

437

438

439

440

441

442

443

444

445

446

447

448

449

450

451

452

evaluate several candidate mechanisms for this difference in fatigue at high contraction velocities.

Although the young women in our study were more physically active than the older women (Table 1), the magnitude of fatigue in each group was nearly identical to that reported by Callahan and Kent-Braun [5], who matched age groups for physical activity and used a similar fatigue protocol to the one used here. Moreover, Englund et al. reported that 12 weeks of progressive resistance training in mobility-limited older men and women improved total torque output (i.e., strength) during a moderate-velocity fatigue protocol of the knee extensors, but had no effect on fatigue, expressed as the relative fall in peak torque [64]. The effects of an intervention designed specifically to increase muscular endurance (i.e., fatigue resistance) remain to be determined, but the results to date suggest that the greater fatigue in older compared with young adults during high-velocity contractions may be independent of physical activity status. In further support of this notion, we observed no relationship between physical activity counts or MVPA and fatigue in either young or older women.

To evaluate the potential role of changes in the completeness of voluntary activation on the age-related differences in muscle fatigue observed here, we compared the changes in the MVIC:80-Hz torque from baseline to fatigue [56]. There were no changes in this measure from baseline to fatigue in either group, indicating no decrease in voluntary activation with fatigue due to high-velocity contractions, a result similar to that reported previously in men [24]. Recently, transcranial magnetic stimulation and femoral nerve stimulation were used to examine potential neural mechanisms for greater fatigue with age following high-velocity contractions of the knee extensors in men and women [29]. In that study, the authors observed a reduction in voluntary 
activation with fatigue in older, but not young women, but these changes in voluntary activation were small and not associated with fatigue. Rather, the authors found a positive association between fatigue and the decrease in stimulated twitch torque, consistent with an age-related difference in peripheral fatigue due to impairment within the muscle, potentially including failure of ECC or crossbridge function. Indeed, subsequent work by this group has shown a significant association between metabolic by-products $\left(\mathrm{Pi}, \mathrm{H}^{+}, \mathrm{H}_{2} \mathrm{PO}_{4}^{-}\right)$and fatigue during a similar protocol, supporting the notion that age-related differences in muscle fatigue occur to differences in the intracellular milieu (62).

Based upon measures of the m-wave (EMG response to a single electrical stimulus), previous studies of knee extensor muscle fatigue indicate that impaired excitability of the neuromuscular junction or along the sarcolemma are not responsible for the age-related differences observed in fatigue during voluntary dynamic contractions such as used here, including those at high velocities $[24,29]$. Thus, these studies also suggest that fatigue under these conditions originates within the muscle. To determine whether greater fatigue in older compared with young muscle was due to failure of ECC, i.e., from the t-tubules to the crossbridges, we compared changes in the $10: 80-\mathrm{Hz}$ torque ratio from baseline to fatigue [31]. This ratio decreased similarly with fatigue in both groups, indicating a comparable failure of excitation-contraction coupling in both young and older women. Thus, it appears that excitationcontraction coupling failure does not explain the age-group difference in fatigue in this study. Torque relaxation $\left(\mathrm{T}_{1 / 2}\right)$ in response to the $80-\mathrm{Hz}$ tetanic stimulus slowed to a similar extent in young and older women during fatigue, which is consistent with some literature $[33,41]$. In contrast, a greater slowing of twitch $\mathrm{T}_{1 / 2}$ in older compared with young muscle following moderate- and high-velocity, but not slow-velocity contractions of the knee extensors 
476 has been reported [24], with no sex-based differences observed [29]. The slowing of $\mathrm{T}_{1 / 2}$ with

477 fatigue is likely due to an accumulation of proton in the cytosol during contractions [43], which

478 has been shown to inhibit $\mathrm{Ca}^{2+}$-ATPase 2 -fold with a change in $\mathrm{pH}$ from 7.1 to 6.6 [65].

479 Indeed, recent work has shown the greater fatigue in older compared with young adults is due to more accumulation of $\mathrm{Pi}$ and $\mathrm{H}^{+}$in the knee extensor muscles of older adults [44].

Notably, baseline $T_{1 / 2}$ was predictive of fatigue in older, but not young women, such that $\sim 60 \%$ of the variation in fatigue during high-velocity contractions in the older group was associated with the rate of torque relaxation in the unfatigued muscle. Whether this relationship may reflect an underlying alteration to contractile function that is protective against the greater fatigue in older muscles during high-velocity contractions, is not known at present. The

486 mechanisms for slowed $T_{1 / 2}$ in old age are not entirely clear, but do not appear to include 487 impaired calcium resequestration by the sarcoplasmic reticulum [61]. Theoretically, the age488 related slowing of $\mathrm{T}_{1 / 2}$ could be caused by: 1) a relatively greater volume of type 1 muscle fibers, 2) slowed detachment of myosin from actin, and 3) a less stiff muscle-tendon unit. These potential mechanisms require further evaluation in the context of muscle fatigue in aging. Recovery from fatigue. Although peak MVDC torque and maximal isometric torque

493 values by the fifth minute of recovery (Fig 3). In contrast to our observation of maximal 494 isometric torque recovering within $5 \mathrm{~min}$, Allman and Rice found incomplete recovery of 495 maximal isometric force of the elbow flexor muscles in young and older men following intermittent, submaximal isometric contractions, which likewise was accompanied by low-

497 frequency fatigue in both groups [32]. More recently, Kent-Braun et al. reported no age-related 498 differences in the recovery of torque following 4-min of contractions of the knee extensors at 
$120^{\circ} \cdot \mathrm{s}^{-1}[66]$. Those authors also observed maximal torque was almost completely recovered

500 within 5 min, consistent with our observations here. Overall, the rapid and complete recovery of

501 MVDC and isometric torque following a high-velocity protocol that induced substantial

502 muscular fatigue suggests a robust resilience of the neuromuscular system in this group of

503 healthy but sedentary older women.

504 Conclusions. This study shows that the greater muscle fatigue observed in older

505 compared with young muscle during high-velocity contractions was not related to differences in

506 muscle architecture, deficits in voluntary activation, failure of excitation-contraction coupling, or

507 an exaggerated, fatigue-induced slowing of contractile properties. We have shown that slower

508 torque relaxation at baseline is inversely associated with greater muscle fatigue in older women,

509 which might be explained by selective atrophy of fast-twitch muscle fibers, slowed crossbridge

510 kinetics, or changes in muscle-tendon unit stiffness with age. Overall, our results suggest that

511 the cause of age-related differences in muscle fatigue reside within the myofibers in healthy

512 older adults; recent work suggests that intracellular metabolism may be important in this process

513 [44]. 
514 Acknowledgements. The authors thank the participants for their contributions to this study. We

515 are also grateful to Dr. Graham E. Caldwell for his insight and discussion relating to the

516 acquisition and analysis of the ultrasound images. Lastly, we thank Sydney L. Connor and Jamie

517 M. Truax for their help with the data analysis.

518 Funding: The authors received no specific funding for this work. 


\section{REFERENCES}

520

521

522

523

524

525

526

527

528

529

530

531

532

533

534

535

536

537

538

539

540

541

542

543

544

545

546

547

548

549
1. Lanza I, Towse T, Caldwell G, Wigmore D, Kent-Braun J. Effects of age on human muscle torque, velocity, and power in two muscle groups. J Appl Physiol. 2003;95: 2361-2369.

2. Valour D, Ochala J, Ballay Y, Pousson M. The influence of ageing on the force-velocitypower characteristics of human elbow flexor muscles. Exp Gerontol. 2003;38: 387-395.

3. Petrella JK, Kim JS, Tuggle SC, Hall SR, Bamman MM. Age differences in knee extension power, contractile velocity, and fatigability. J Appl Physiol. 2005;98: 211-220.

4. Thom JM, Morse CI, Birch KM, Narici MV. Influence of muscle architecture on the torque and power-velocity characteristics of young and elderly men. Eur J Appl Physiol. 2007;100: 613619.

5. Callahan D, Kent-Braun J. Effect of old age on human skeletal muscle force-velocity and fatigue properties. J Appl Physiol. 2011;111: 1345-1352.

6. McNeil CJ, Vandervoort AA, Rice CL. Peripheral impairments cause a progressive agerelated loss of strength and velocity-dependent power in the dorsiflexors. J Appl Physiol. 2007;102(5): 1962-1968.

7. Kent-Braun J, Ng A, Young K. Skeletal muscle contractile and noncontractile components in young and older women and men. J Appl Physiol. 2000;88: 662-668.

8. Hogrel J, Barnouin Y, Azzabou N, Butler-Browne G, Voit T, Moraux A, et al. NMR imaging estimates of muscle volume and intramuscular fat infiltration in the thigh: variations with muscle, gender, and age. Age. 2015;37(3): 9798.

9. Lexell J. Human aging, muscle mass, and fiber type composition. J Gerontol A Biol Sci Med Sci. 1995;50: 11-16.

10. Trappe T, Lindquist D, Carrithers J. Muscle-specific atrophy of the quadriceps femoris with aging. J Appl Physiol. 2001;90: 2070-2074.

11. Dalton B, Power G, Vandervoort A, Rice C. Power loss is greater in old men than young men during fast plantar flexion contractions. J Appl Physiol. 2010;109: 1441-1447.

12. Kamen G, Sison S, Du C, Patten C. Motor unit discharge behavior in older adults during maximal-effort contractions. J Appl Physiol. 1995;79: 1908-1913.

13. Mayson DJ, Kiely DK, LaRose SI, Bean JF. Leg strength or velocity of movement: which is more influential on the balance of mobility limited elders? Am J Phys Med Rehabil. 2008;87: 969-976. 
14. Bean JF, Kiely DK, LaRose S, Leveille SG. Which impairments are most associated with high mobility performance in older adults? Implications for a rehabilitation prescription. Arch Phys Med Rehabil. 2008;89: 2278-2284.

15. Cuoco A, Callahan DM, Sayers S, Frontera WR, Bean J, Fielding RA. Impact of muscle power and force on gait speed in disabled older men and women. J Gerontol A Biol Sci Med Sci. 2004;59: 1200-1206.

16. Madigan ML. Age-related differences in muscle power during single-step balance recovery. J Appl Biomech. 2006;22(3): 186-193.

17. Lieber R, Fridén J. Functional and clinical significance of skeletal muscle architecture. Muscle Nerve. 2000;23: 1647-1666.

18. Blazevich AJ. Effects of physical training and detraining, immobilisation, growth and aging on human fascicle geometry. Sports Med. 2006;36: 1003-1018.

19. Spector SA, Gardiner PF, Zernicke RF, Roy RR, Edgerton VR. Muscle architecture and force-velocity characteristics of cat soleus and medial gastrocnemius: implications for motor control. J Neurophysiol. 1980;44: 951-960.

20. Morse C, Thom J, Birch K, Narici M. Changes in triceps surae muscle architecture with sarcopenia. Acta Physiol Scand. 2005;183(3): 291-298.

21. Narici M, Maganaris C, Reeves N, Capodaglio P. Effect of aging on human muscle architecture. J Appl Physiol. 2003;95(6): 2229-2234.

22. Power G, Makrakos D, Rice C, Vandervoort A. Enhanced force production in old age is not a far stretch: an investigation of residual force enhancement and muscle architecture. Physiol Rep. 2013;1: 12 .

23. Kubo K, Kanehisa H, Azuma K, Ishizu M, Kuno S, Okada M, et al. Muscle architectural characteristics in young and elderly men and women. Int J Sports Med. 2003;24: 125-130.

24. Dalton B, Power G, Vandervoort A, Rice C. The age-related slowing of voluntary shortening velocity exacerbates power loss during repeated fast knee extensions. Exp Gerontol. 2012;47: 85-92.

25. Dalton B, Power G, Paturel J, Rice C. Older men are more fatigable than young when matched for maximal power and knee extension angular velocity is unconstrained. Age. 2015;37: $1-16$.

26. Wallace J, Power G, Rice C, Dalton B. Time-dependent neuromuscular parameters in the plantar flexors support greater fatigability of old compared with younger males. Exp Gerontol. 2016;74: 13-20. 
27. Kent-Braun J, Fitts R, Christie A. Skeletal muscle fatigue. Compr Physiol. 2012;2: 997-1044.

28. Christie A, Snook E, Kent-Braun J. Systematic review and meta-analysis of skeletal muscle fatigue in old age. Med Sci Sports Exerc. 2011;43: 568-577.

29. Sundberg CW, Kuplic A, Hassanlouei H, Hunter SK. Mechanisms for the age-related increase in fatigability of the knee extensors in old and very old adults. J Appl Physiol. 2018;125: 146-158.

30. Kent-Braun J. Central and peripheral contributions to muscle fatigue in humans during sustained maximal effort. Eur J Appl Physiol Occup Physiol. 1999;80: 57-63.

31. Edwards RH, Hill DK, Jones DA, Merton PA. Fatigue of long duration in human skeletal muscle after exercise. J Physiol. 1977;272: 769-778.

32. Allman B, Rice C. Incomplete recovery of voluntary isometric force after fatigue is not affected by old age. Muscle Nerve. 2001;24: 1156-1167.

33. Chung L, Callahan D, Kent-Braun J. Age-related resistance to skeletal muscle fatigue is preserved during ischemia. J Appl Physiol. 2007;103: 1628-1635.

34. Power G, Dalton B, Rice C, Vandervoort A. Power loss is greater following lengthening contractions in old versus young women. Age. 2012;34: 737-750.

35. Chin ER, Balnave CD, Allen DG. Role of intracellular calcium and metabolites in lowfrequency fatigue of mouse skeletal muscle. Am J Physiol. 1997;272: C550-9.

36. Westerblad H, Duty S, Allen DG. Intracellular calcium concentration during low-frequency fatigue in isolated single fibers of mouse skeletal muscle. J Appl Physiol. 1993;75: 382-388.

37. Jiménez-Moreno R, Wang Z, Gerring RC, Delbono O. Sarcoplasmic reticulum Ca2 release declines in muscle fibers from aging mice. Biophys J. 2008;94: 3178-3188.

38. Kent-Braun J, Ng A, Doyle J, Towse T. Human skeletal muscle responses vary with age and gender during fatigue due to incremental isometric exercise. J Appl Physiol. 2002;93: 18131823.

39. Lexell J, Taylor C, Sjöström M. What is the cause of the ageing atrophy?: Total number, size and proportion of different fiber types studied in whole vastus lateralis muscle from 15-to 83year-old men. J Neurol Sci. 1988;84: 275-294.

40. Miller M, Bedrin N, Callahan D, Previs M, Jennings II M, Ades P, et al. Age-related slowing of myosin actin cross-bridge kinetics is sex specific and predicts decrements in whole skeletal muscle performance in humans. J Appl Physiol. 2013;115(7): 1004-1014. 
41. Lanza I, Russ D, Kent-Braun J. Age-related enhancement of fatigue resistance is evident in men during both isometric and dynamic tasks. J Appl Physiol. 2004;97: 967-975.

42. Allen D, Lamb G, Westerblad H. Skeletal muscle fatigue: cellular mechanisms. Physiol Rev. 2008;88: 287-332.

43. Cady EB, Elshove H, Jones DA, Moll A. The metabolic causes of slow relaxation in fatigued human skeletal muscle. J Physiol. 1989;418: 327-337.

44. Sundberg CW, Prost RW, Fitts RH, Hunter SK. Bioenergetic basis for the increased fatigability with ageing. J Physiol. 2019;597: 4943-4957.

45. Faul F, Erdfelder E, Lang A, Buchner A. G* Power 3: A flexible statistical power analysis program for the social, behavioral, and biomedical sciences. Behav Res Methods. 2007;39: 175191.

46. Thomas S, Reading J, Shephard RJ. Revision of the Physical Activity Readiness Questionnaire (PAR-Q). Can J Sport Sci. 1992;17: 338-345.

47. Sarwar R, Niclos BB, Rutherford OM. Changes in muscle strength, relaxation rate and fatiguability during the human menstrual cycle. J Physiol. 1996;493: 267-272.

48. Podsiadlo D, Richardson S. The timed "Up \& Go": a test of basic functional mobility for frail elderly persons. J Am Geriatr Soc. 1991;39: 142-148.

49. Simonsick EM, Newman AB, Nevitt MC, Kritchevsky SB, Ferrucci L, Guralnik JM, et al. Measuring higher level physical function in well-functioning older adults: expanding familiar approaches in the Health ABC study. J Gerontol A Biol Sci Med Sci. 2001;56: 644-649.

50. Matthews CE, Ainsworth BE, Thompson RW, Bassett DR,Jr. Sources of variance in daily physical activity levels as measured by an accelerometer. Med Sci Sports Exerc. 2002;34(8): 1376-1381.

51. Matthews CE, Chen KY, Freedson PS, Buchowski MS, Beech BM, Pate RR, et al. Amount of time spent in sedentary behaviors in the United States, 2003-2004. Am J Epidemiol. 2008;167(7): 875-881.

52. Freedson PS, Melanson E, Sirard J. Calibration of the Computer Science and Applications, Inc. accelerometer. Med Sci Sports Exerc. 1998;30: 777-781.

53. Murach K, Greever C, Luden ND. Skeletal muscle architectural adaptations to marathon run training. Applied Physiology, Nutrition, and Metabolism. 2014;40(1): 99-102.

54. Reeves ND, Narici MV. Behavior of human muscle fascicles during shortening and lengthening contractions in vivo. J Appl Physiol. 2003;95: 1090-1096. 
55. Callahan D, Foulis S, Kent-Braun J. Age-related fatigue resistance in the knee extensor muscles is specific to contraction mode. Muscle Nerve. 2009;39: 692-702.

56. Bigland-Ritchie BR, Jones DA, Hosking GP, Edwards RH. Central and peripheral fatigue in sustained maximum voluntary contractions of human quadriceps muscle. Clin Sci Mol Med. 1978;54: 609-614.

57. Cohen J. Statistical power analysis for the behavioral sciences. New York, NY: Routledge Academic; 1988.

58. Kubo K, Kanehisa H, Azuma K, Ishizu M, Kuno SY, Okada M, et al. Muscle architectural characteristics in women aged 20-79 years. Med Sci Sports Exerc. 2003;35: 39-44.

59. Allman B, Rice C. An age-related shift in the force-frequency relationship affects quadriceps fatigability in old adults. J Appl Physiol. 2004;96: 1026-1032.

60. Kubo K, Kanehisa H, Miyatani M, Tachi M, Fukunaga T. Effect of low-load resistance training on the tendon properties in middle-aged and elderly women. Acta Physiol Scand. 2003;178: 25-32.

61. Hunter SK, Thompson MW, Ruell PA, Harmer AR, Thom JM, Gwinn TH, et al. Human skeletal sarcoplasmic reticulum $\mathrm{Ca} 2+$ uptake and muscle function with aging and strength training. J Appl Physiol. 1999;86: 1858-1865.

62. Lanza I, Befroy D, Kent-Braun J. Age-related changes in ATP-producing pathways in human skeletal muscle in vivo. J Appl Physiol. 2005;99: 1736-1744.

63. Callahan D, Umberger B, Kent J. Mechanisms of in vivo muscle fatigue in humans: Investigating age-related fatigue resistance with a computational model. J Physiol. 2016;594(12): 3407-3421.

64. Englund DA, Price LL, Grosicki GJ, Iwai M, Kashiwa M, Liu C, et al. Progressive resistance training improves torque capacity and strength in mobility-limited older adults. J Gerontol A Biol Sci Med Sci. 2018;74(8): 1316-1321.

65. Allen D, Lannergren J, Westerblad H. Muscle cell function during prolonged activity: cellular mechanisms of fatigue. Exp Physiol. 1995;80: 497-527.

66. Kent-Braun JA, Callahan DM, Fay JL, Foulis SA, Buonaccorsi JP. Muscle weakness, fatigue, and torque variability: effects of age and mobility status. Muscle Nerve. 2014;49: 209217. 


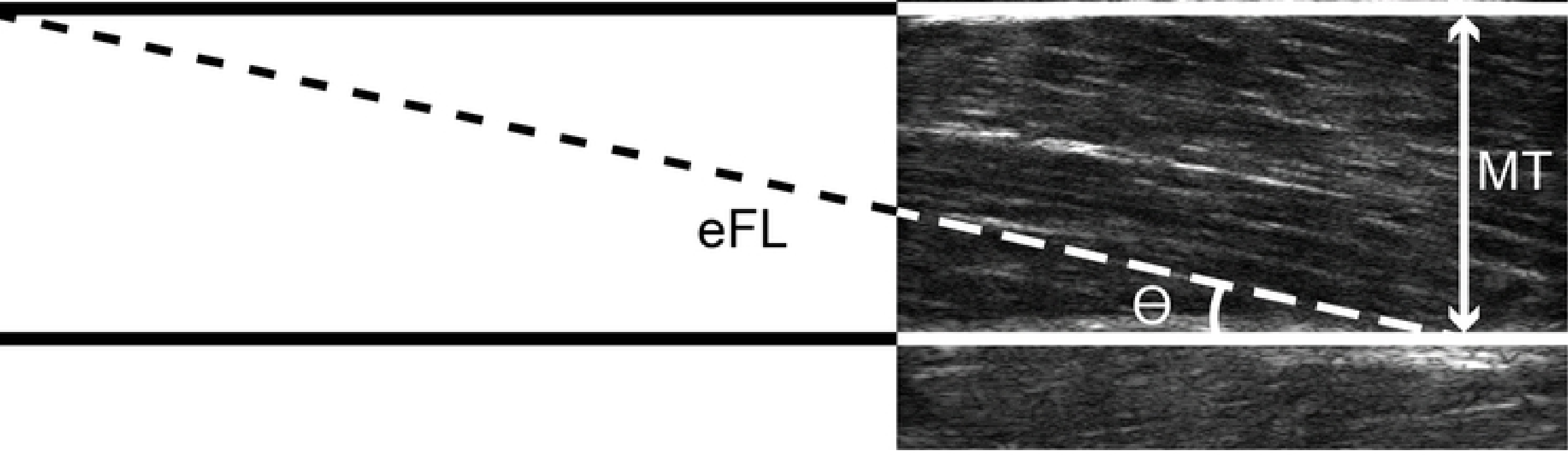

Fig 1 


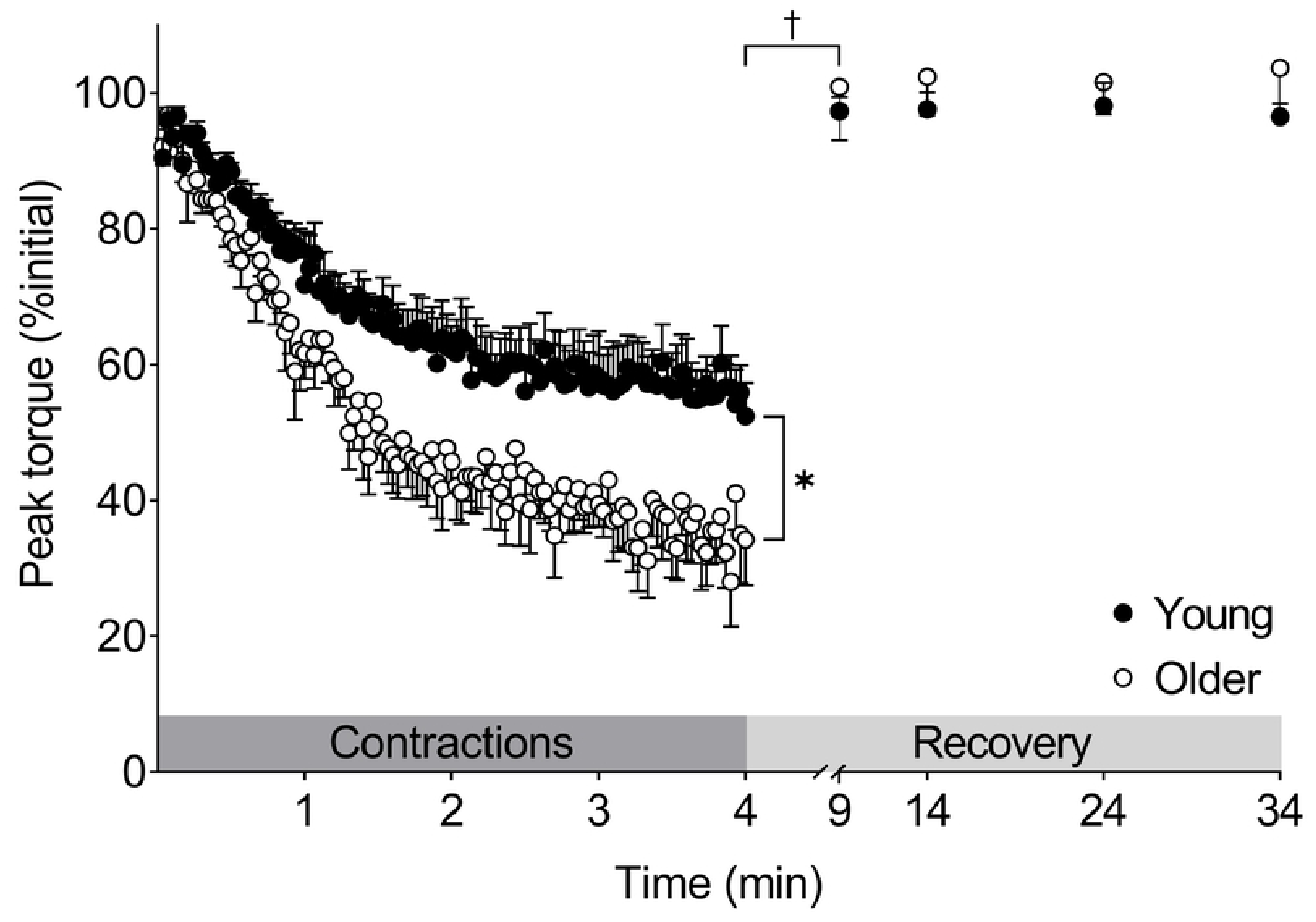

Fig 3 
A

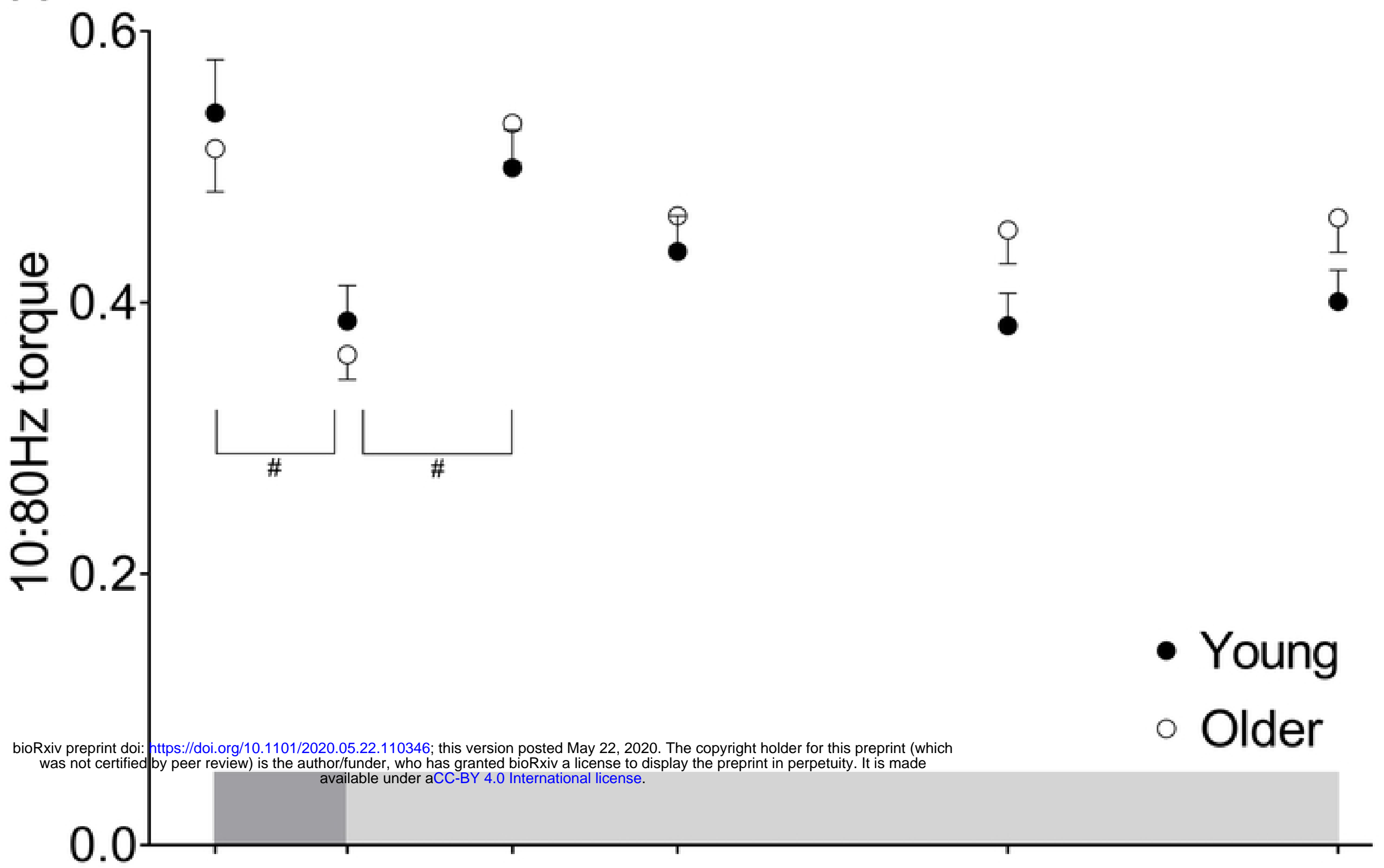

B

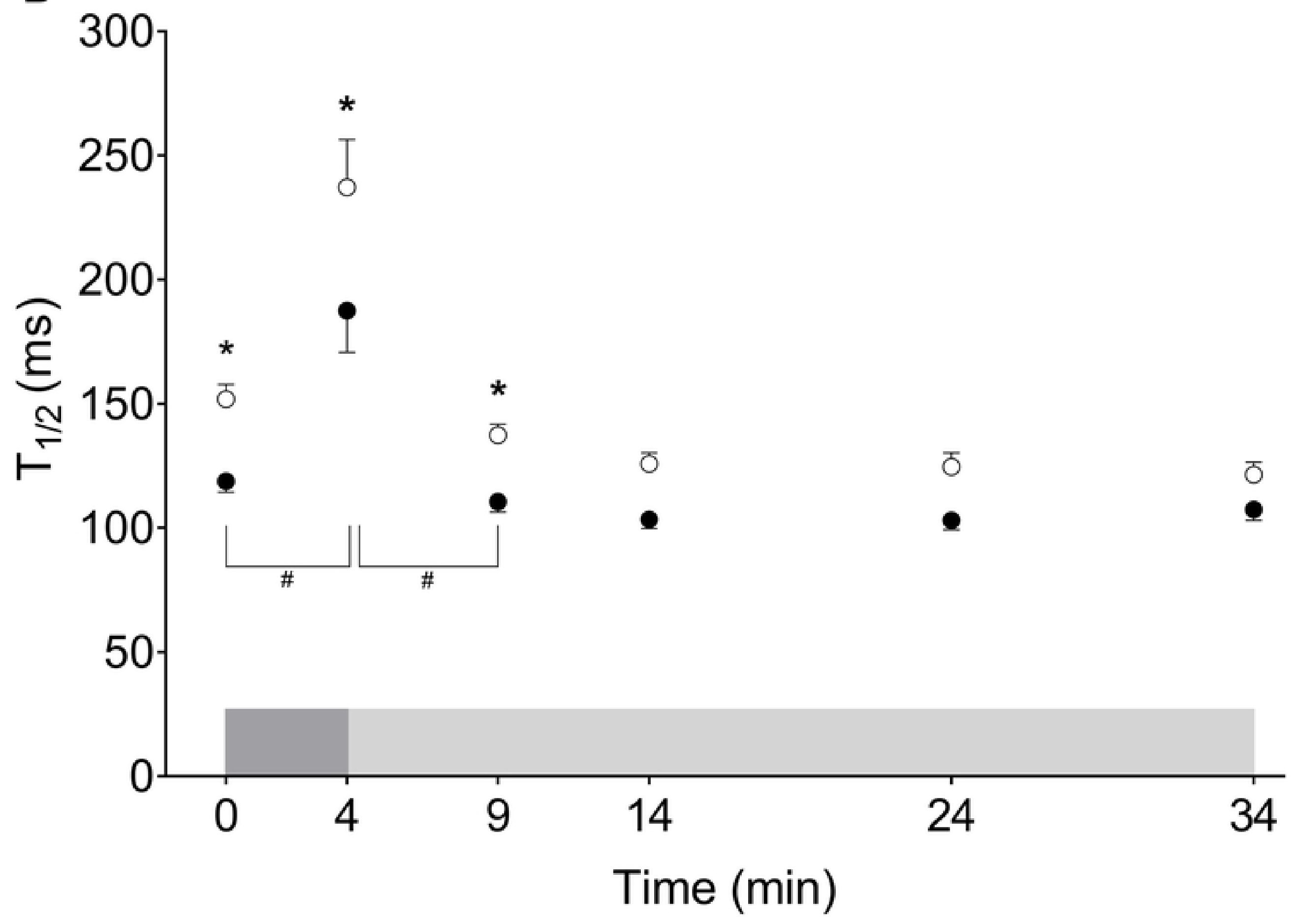

Fig 4 


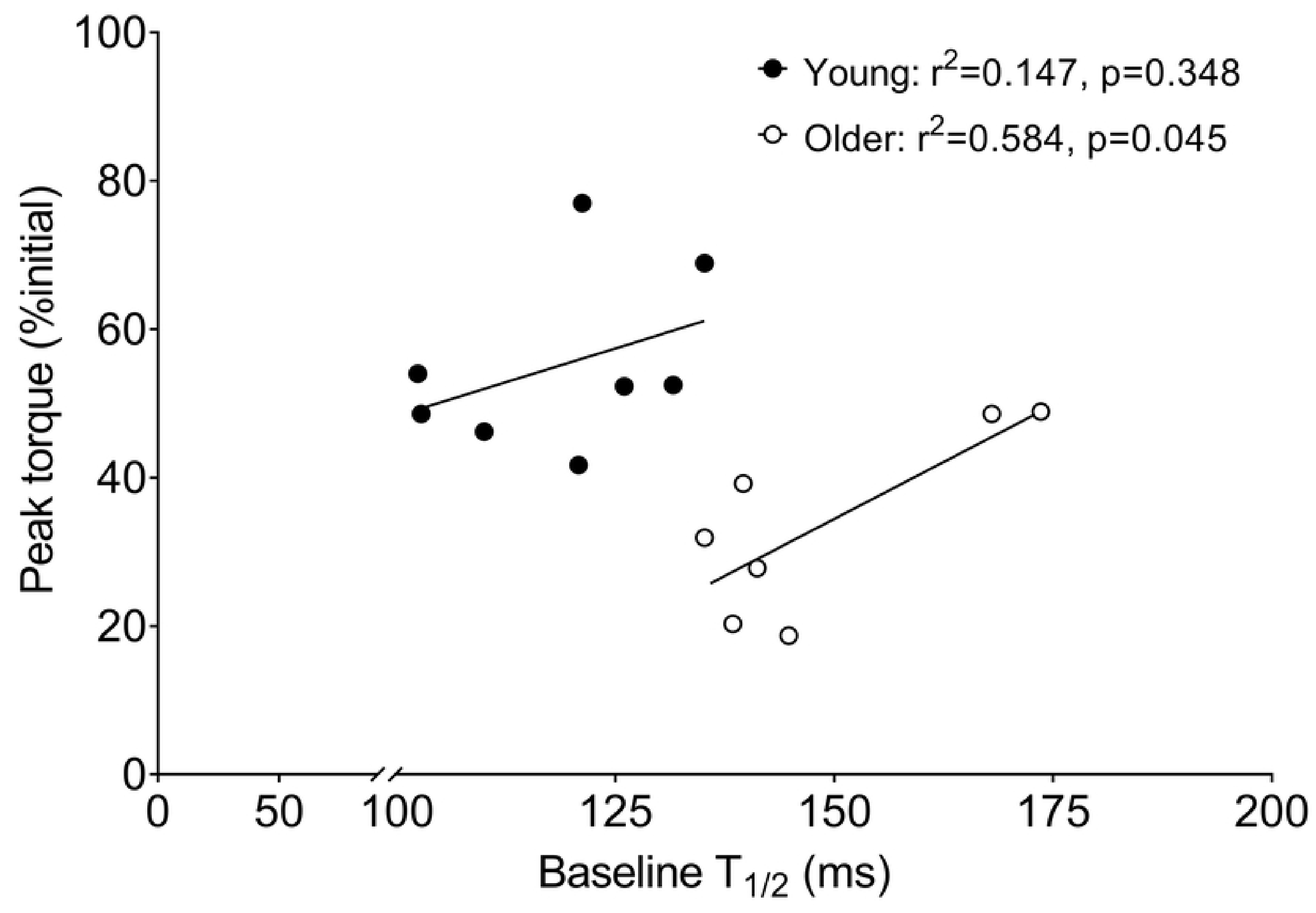

Fig 5 